\title{
Nurse-led Care and Patients as Partners Are Essential Aspects of the Future of Rheumatology Care
}

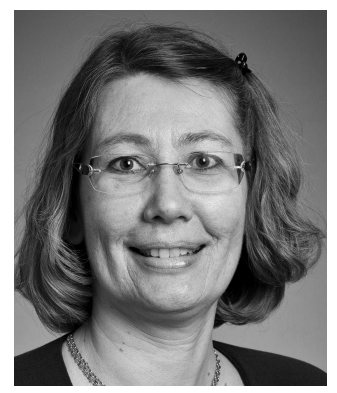

Living with chronic inflammatory arthritis (CIA), such as rheumatoid arthritis (RA) or spondyloarthritis (SpA), affects not only patients' physical functioning but also emotional, psychological, and social aspects that have a global effect on their life situation as a whole ${ }^{1}$. The multidisciplinary team is important for the rheumatology care of patients with CIA, which should be delivered with an awareness of the patients' whole life situation. The team should enable these patients to care for themselves and retain or regain optimum independence. The various professional categories in the team have distinct roles but collaborate to focus on the patients' resources and needs ${ }^{2}$. Recommendations for rheumatology nursing management of CIA from the European League Against Rheumatism state that rheumatology nurses should participate in comprehensive disease management to control disease activity, as well as in identifying, assessing, and addressing psychosocial issues. This work is a valuable complement to the medical care and helps lower healthcare costs. For patients to achieve a greater sense of control, self-efficacy and empowerment, the nurse should meet the patient's expressed needs and promote self-management skills ${ }^{3}$.

Rheumatology research has completely changed the therapeutic arena over the past 2 decades, generating the development of the biological disease-modifying antirheumatic drugs (bDMARD) for a greater number of indications ${ }^{4}$. Despite this advance, research on nurse-led rheumatology care (NLC) has predominantly focused on patients with RA and conventional DMARD. A systematic review, including 4 randomized controlled trials (RCT) from 1994 to 2006, revealed in a metaanalysis $(n=431)$ that NLC compared to rheumatologist-led care (RLC) added value by improving patients' perceived quality of life and knowledge, and lessening fatigue. While patient-reported outcomes such as functional status, satisfaction, pain, stiffness, and coping with arthritis favored NLC, there was insufficient evidence to draw conclusions ${ }^{5}$.

Subsequent comprehensive RCT $(\mathrm{n}=287 \text { and } \mathrm{n}=181)^{6,7}$ showed that NLC was effective compared to RLC in improvement in self-efficacy, confidence, satisfaction ${ }^{6}$, pain, and physical function ${ }^{7}$. NLC was safe without deterioration in disease activity 6,7 and generally had lower costs, but was not significantly cost-effective $\mathrm{e}^{7,8}$. An observational nonrandomized study $(n=349)$ showed that NLC in general practices were not significantly cost-effective ${ }^{9}$.

In this issue of The Journal, Garner, et al, in a systematic review, included data from RCT, observational nonrandomized studies, and qualitative studies on NLC for patients with $\mathrm{RA}^{10}$. The authors comprehensively assess the effect of NLC on quality of care using as a framework the Alberta Quality Matrix for Health, which included 6 quality dimensions: acceptability, accessibility, appropriateness, effectiveness, efficiency, and safety ${ }^{11}$. The dimension "acceptability" focuses on patient-centered care respecting the patients' needs, preferences, and expectations and participation in their own care. Garner, et al used the outcome measures satisfaction and clinical attendance: they concluded that NLC was acceptable compared to other models. The dimension "accessibility" refers to patients' access to care in terms of a suitable setting within a reasonable time and distance. Garner, et al used outcome measures: access to care and continuity of care. They described that patients experienced accessibility, continuity, and sufficient time in NLC, but concluded that the current evidence was insufficient, because only qualitative studies reported on access to NLC. The dimension "appropriateness" refers to the collaboration between the patient and the healthcare provider in balancing patients' needs and preferences with evidence-based practice. Garner, et al used these outcome measures: relationship with care provider, holistic care, and provision of information. Patients experienced the relationship, holistic person-centered care, nurses' competence, and that NLC improved their knowledge and skills through patient education. The conclusion was that NLC appears to be appropriate from the patient perspective, but few quantitative measures were reported. The dimension "effectiveness"

\section{See Nurse-led care in RA, page 757}


refers to the efficacy of the intervention to provide the best outcome for the patient based on scientific knowledge. Garner, et al used the outcome measures 28-joint Disease Active Score, Health Assessment Questionnaire, pain, fatigue, morning stiffness, Arthritis Impact Measurement Scale, and self-efficacy. In these outcome measures NLC was equal or superior in comparison with other models, with the conclusion that NLC was effective. The dimension "efficiency" focuses on how resources are optimally used for the patients. Garner, et al used as outcome measures the number of conferrals and referrals, appointment length, and costs. Most studies suggested that NLC was equal in costs or less costly than other models, but the authors conclude that more comprehensive studies about cost-effectiveness are required. The last dimension, "safety," focuses on actions to minimize risks or harmful results. Garner, et al used the outcome measures out-of-range blood test, adherence to required monitoring, healthcare contacts, hospitalizations, and death. In these outcome measures NLC was equal compared to other models, with the conclusion that NLC was safe $^{10}$.

Both qualitative and quantitative approaches are used in the systematic review to provide a more comprehensive and thorough understanding of the quality of $\mathrm{NLC}^{10}$. Although qualitative studies were included in the systematic review, only the dimensions containing quantitative outcome measures were considered to contribute to evidence. To understand the complexity of rheumatology care, research must be based on both the positivistic paradigm, quantitative research, where reality is objective and generalizable and on the naturalistic paradigm, qualitative research, where reality is multiple and subjective. Qualitative methods should be considered to improve the understanding of rheumatic diseases and rheumatology care ${ }^{12}$. The 6 dimensions in the framework Alberta Quality Matrix for Health are based on values from a person-centered care (PCC) approach with focus on patients' needs, preferences, and expectations. The framework emphasizes patient participation, with collaboration between patient and healthcare provider to facilitate the optimal outcome for the patients ${ }^{11}$.

In the management of patients with CIA, rheumatology care has changed from a more basic to an advanced level, in line with the development of DMARD treatment and biological therapy. The rheumatology nurse's role has developed from that of an expert advisor to a partner, where patients are necessary and equal participants. Rheumatology care is intended to meet patients' individual needs and improve patient care $^{3}$, which can be achieved with a PCC approach, meaning holistic, respectful, individualized, and empowering care ${ }^{13}$. PCC involves creating a partnership characterized by shared information, deliberation, and decision making based on patients' narrative. Patients are seen as capable persons who can talk about and assume responsibility for their actions. In PCC the patient and the healthcare provider are seen as equals in a partnership with a mutual respect for each other's knowledge. PCC emphasizes the importance of knowing the person behind the patient and treating her/him as a unique individual with her/his own will, emotions, needs, and resources, recognizing the person as an expert on her/his illness and life situation ${ }^{14}$. In an RCT including patients with CIA with low disease activity or in remission who were undergoing biological therapy, NLC based on PCC was designed ${ }^{15}$. Replacing 1 of the 2 annual rheumatologist outpatient monitoring visits with an NLC monitoring visit based on PCC was found to be safe and effective ${ }^{15,16}$. Patients experienced that the NLC, based on PCC, added value to followup care by providing a sense of security, familiarity, and participation. The patients perceived that NLC complemented RLC, and PCC added a new dimension. The rheumatology care became more optimal when patients were given the opportunity to see several professionals on a regular basis ${ }^{17}$. A paradigm shift is taking place in rheumatology care and a PCC approach is gradually being integrated into daily care routines ${ }^{18}$.

Because time and money are lacking in clinical practice, it is important to involve patients in their care and use their resources in the best possible way. An NLC based on PCC is one way of achieving this objective. PCC is designed to encourage and empower patients to play an active role in their care and treatment. Healthcare services providing PCC and keeping the patients' resources and needs in focus are important during the patient's healthcare journey. A rheumatology nurse who uses PCC can be key to reducing costs without affecting the quality of care. Patients with stable CIA undergoing biological therapy can be monitored with reduced resource use and lower annual costs with NLC based on $\mathrm{PCC}^{16}$ compared to an RLC, with no difference in clinical outcomes ${ }^{15}$.

The question has been raised of whether mid-level providers such as rheumatology nurses or physician assistants are able to alleviate the shortages of rheumatologists ${ }^{19}$. Interventions with mid-level providers based on PCC could free rheumatologists for more intensive monitoring of patients early in the course of the disease or patients with high disease activity. Research supports the implementation of PCC in rheumatology care of patients with CIA with low disease activity or remission ${ }^{20}$. Further research is needed to implement and evaluate the longterm effect of NLC or physician assistant clinics, based on PCC for monitoring therapies in patients with CIA.

INGRID LARSSON, RN, PhD,

School of Health and Welfare, Halmstad University, and Spenshult Research and Development Center, Halmstad, Sweden.

Address correspondence to I. Larsson, School of Health and Welfare, Halmstad University Box 823, S-30118 Halmstad, Sweden.

E-mail: Ingrid.larsson@hh.se

Personal non-commercial use only. The Journal of Rheumatology Copyright @ 2017 . All rights reserved. 


\section{REFERENCES}

1. Hewlett S, Chalder T, Choy E, Cramp F, Davis B, Dures E, et al. Fatigue in rheumatoid arthritis: time for a conceptual model. Rheumatology 2011;50:1004-6.

2. Walker J. Rheumatoid arthritis: role of the nurse and multidisciplinary team. Br J Nurs 2012;21:334-6, 339.

3. van Eijk-Hustings Y, van Tubergen A, Bostrom C, Braychenko E, Buss B, Felix J, et al. EULAR recommendations for the role of the nurse in the management of chronic inflammatory arthritis. Ann Rheum Dis 2012;71:13-9.

4. Furst DE, Keystone EC, So AK, Braun J, Breedveld FC, Burmester GR, et al. Updated consensus statement on biological agents for the treatment of rheumatic diseases, 2012. Ann Rheum Dis 2013;72 Suppl 2:ii2-34.

5. Ndosi M, Vinall K, Hale C, Bird H, Hill J. The effectiveness of nurse-led care in people with rheumatoid arthritis: a systematic review. Int J Nursing Studies 2011;48:642-54.

6. Primdahl J, Sorensen J, Horn HC, Petersen R, Horslev-Petersen K. Shared care or nursing consultations as an alternative to rheumatologist follow-up for rheumatoid arthritis outpatients with low disease activity - patient outcomes from a 2-year, randomised controlled trial. Ann Rheum Dis 2014;73:357-64.

7. Ndosi M, Lewis M, Hale C, Quinn H, Ryan S, Emery P, et al. The outcome and cost-effectiveness of nurse-led care in people with rheumatoid arthritis: a multicentre randomised controlled trial. Ann Rheum Dis 2014;73:1975-82.

8. Sorensen J, Primdahl J, Horn H, Horslev-Petersen K. Shared care or nurse consultations as an alternative to rheumatologist follow-up for rheumatoid arthritis (RA) outpatients with stable low disease-activity RA: cost-effectiveness based on a 2-year randomized trial. Scand J Rheumatol 2015;44:13-21.

9. Watts RA, Mooney J, Barton G, MacGregor AJ, Shepstone L, Irvine $\mathrm{L}$, et al. The outcome and cost-effectiveness of nurse-led care in the community for people with rheumatoid arthritis: a non-randomised pragmatic study. BMJ Open 2015;5:e07696.

10. Garner S, Lopatina E, Rankin JA, Marshall DA. Nurse-led care for patients with rheumatoid arthritis: a systematic review of the effect of quality of care. J Rheumatol 2017;44:757-65.
11. Alberta Quality Matrix for Health. Health Quality Council of Alberta; 2005 [updated 2005; Internet. Accessed February 26, 2017.] Available from: https://d10k7k7mywg42z.cloudfront.net/ assets/56a00bd2d4c9612e3610b6ce/HQCA_11x8_5_Matrix.pdf

12. Johnson SR, O'Brien KK. Qualitative methods in systemic sclerosis research. J Rheumatol 2016;43:1265-7.

13. Morgan S, Yoder LH. A concept analysis of person-centered care. J Holist Nurse 2012;30:6-15.

14. Ekman I, Swedberg K, Taft C, Lindseth A, Norberg A, Brink E, et al. Person-centered care-ready for prime time. Eur J Cardiovasc Nurs 2011;10:248-51.

15. Larsson I, Fridlund B, Arvidsson B, Teleman A, Bergman S. Randomized controlled trial of a nurse-led rheumatology clinic for monitoring biological therapy. J Advanced Nursing 2014;70:164-75

16. Larsson I, Fridlund B, Arvidsson B, Teleman A, Svedberg P, Bergman S. A nurse-led rheumatology clinic versus rheumatologist-led clinic in monitoring of patients with chronic inflammatory arthritis undergoing biological therapy: a cost comparison study in a randomised controlled trial. BMC Musculoskelet Disord 2015;16:354.

17. Larsson I, Bergman S, Fridlund B, Arvidsson B. Patients' experiences of a nurse-led rheumatology clinic in Sweden: a qualitative study. Nurs Health Sci 2012;14:501-7.

18. Bala SV, Samuelson K, Hagell P, Svensson B, Fridlund B, Hesselgard K. The experience of care at nurse-led rheumatology clinics. Musculoskeletal Care 2012;10:202-11.

19. Solomon DH, Bitton A, Fraenkel L, Brown E, Tsao P, Katz JN. Roles of nurse practitioners and physician assistants in rheumatology practices in the US. Arthritis Care Res 2014; 66:1108-13.

20. Larsson I. Person-centred care in rheumatology nursing in patients undergoing biological therapy: an explorative and interventional study [PhD thesis]. Jönköping University; 2013.

J Rheumatol 2017;44:720-2; doi:10.3899/jrheum.170215 